\title{
Lens-less Fourier Transform Holography for Electron Vortex Beams
}

Ken Harada $^{1}$, Yoshimasa Ono ${ }^{1}$ and Yoshio Takahashi ${ }^{2}$

${ }^{1}$ RIKEN, Hatoyama, Saitama, Japan, ${ }^{2}$ Hitachi, Ltd., Hatoyama, Saitama, Japan

Electron holography is well known as the most powerful tool for observing amplitude and phase distributions of electron waves. In an ordinary electron holography, electron holograms recorded under an infocus condition for the specimens as image holograms are characterized by specimen images and interference fringes superimposed on the images. Observations and analyses of specimens are performed in the real space. On the other hand, electron holography in the reciprocal space is rare because very few appropriate reference waves in the reciprocal space are found.

Fourier transform holography is one solution to overcome these difficulties, where a reference wave source is positioned in the same plane as that of specimens. Interferograms with the object and reference waves are recorded in the angular patterns generated by these two waves. Since recording interferences between the object and reference waves are realized without using a lens system, this technique is called lens-less Fourier transform holography [1].

Figure 1 shows a schematic diagram of the optical system of lens-less Fourier transform holography for electron vortex-beam experiment. By treating Bragg diffraction waves as object waves and a transmitted spherical wave along the optical axis as a reference wave whose source is placed at the reciprocal plane, two-wave interference patterns are recorded away from the reciprocal plane.

Figure 2(a) shows the underfocus hologram of the first order diffraction spots in red broken squares for Figs. 2(b) and (c). Upper parts in (b) and (c) show amplitude images and lower parts show phase images. These amplitude and phase images were reconstructed through only one Fourier transformation. These amplitude images in Fig. 2 correspond to the vortex beams of the first order spots in the reciprocal plane. Lower parts of Figs. 2(b) and (c), show the reconstructed phase distributions of the object images, vortex beams, in fan-like structures. Although not shown in the figures here, the reconstructed phase distributions from the underfocus and overfocus holograms are the same.

In conclusion, we have developed lens-less Fourier transform holography for reconstruction of electron vortex beams in the reciprocal plane. The underfocus and overfocus holograms at displaced positions away from the reciprocal plane are recorded; then both vortex beams are reconstructed in amplitude and phase distributions in terms of object and conjugate images. We have confirmed that the developed lensless Fourier transform holography has an advantage of reconstructing wave properties in the reciprocal space. With this development together with the conventional reconstruction method [2], electron holography can systematically cover the whole space ranges from the real space to the reciprocal space [3]. 


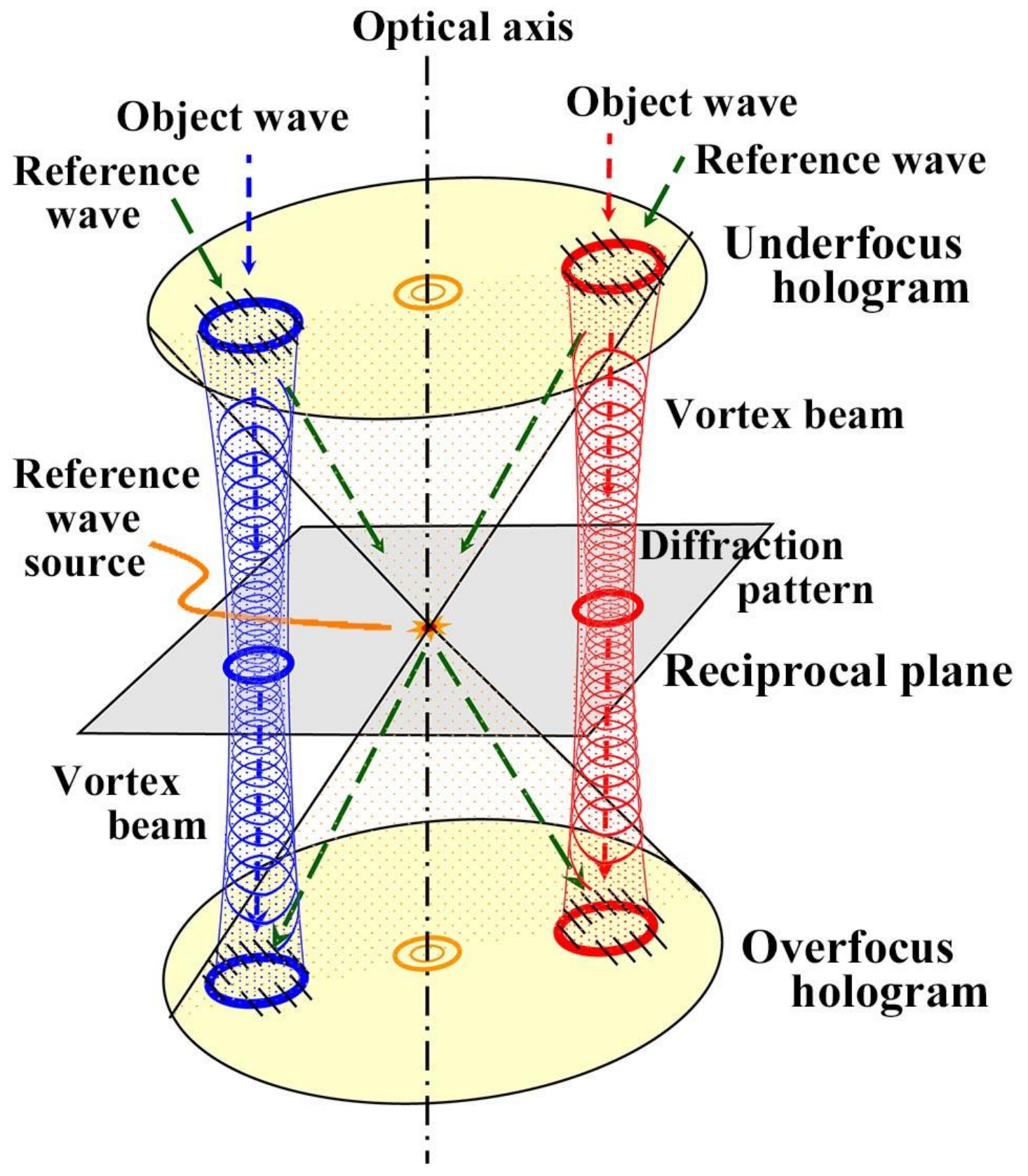

Figure 1. Schematic diagram of the lens-less Fourier transform holography. Two vortex beams (blue and red) and a transmitted wave (green) are superimposed above and below the reciprocal plane as underfocus and overfocus holograms, respectively. 

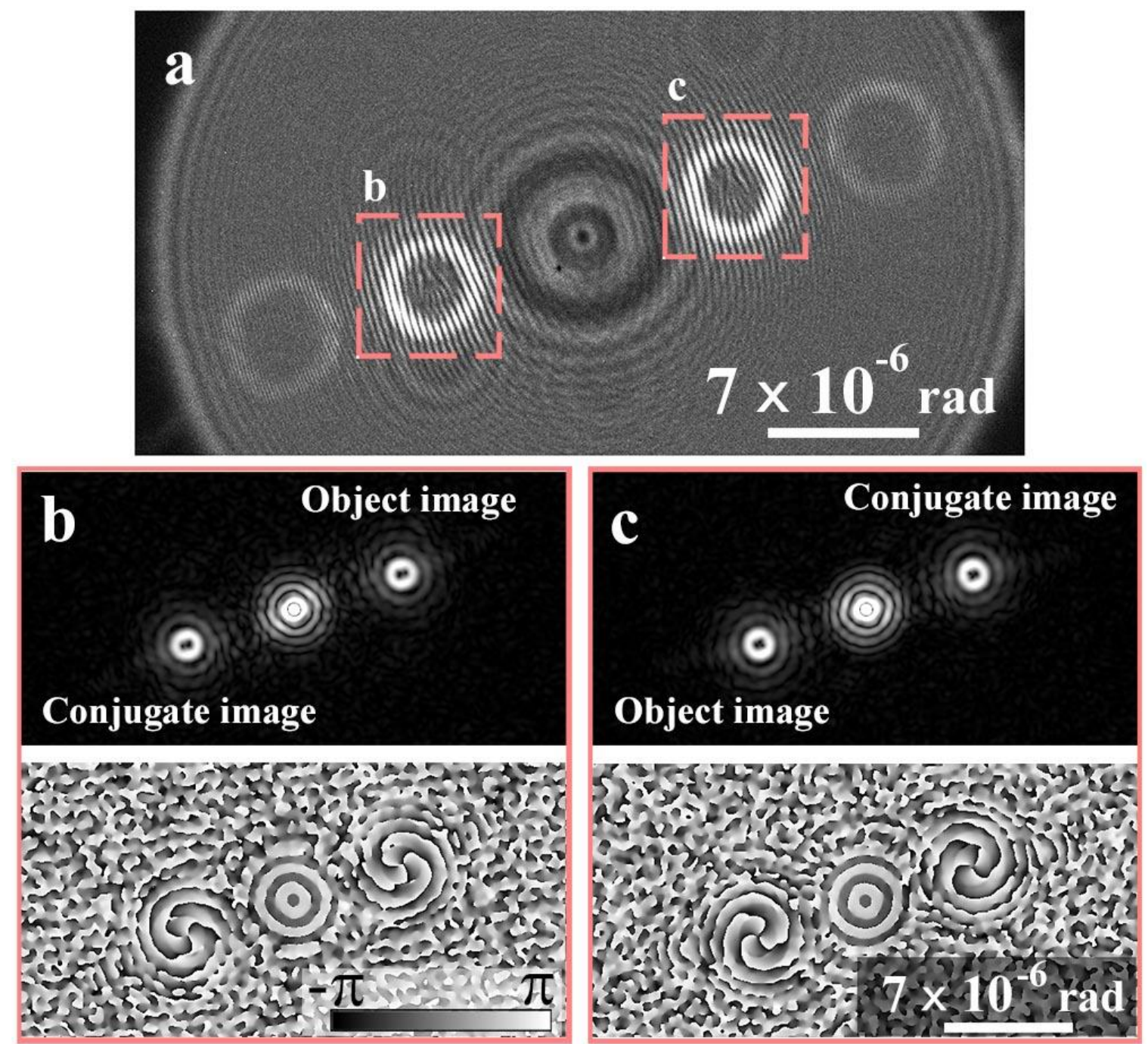

Figure 2. Schematic diagram of the lens-less Fourier transform holography. Two vortex beams (blue and red) and a transmitted wave (green) are superimposed above and below the reciprocal plane as underfocus and overfocus holograms, respectively.

\section{References}

[1] K. Harada et al, Microscopy, 69 (2020), to be published.

[2] K. Harada et al, APEX, 13 (2020), doi.org/10.35848/1882-0786/ab7059.

[3] The authors would like to thank Prof. J. Yamasaki of Osaka University for discussions and Ms. K. Shimada of RIKEN for preparing fork-shaped gratings. This work was supported by KAKENHI, Grantin-Aid for Scientific Research ((B) 18H03475). 